\section{Le problème des opioïdes}

Au Congrès annuel de la SCMI tenu récemment à Toronto, la séance plénière s'intitulait « La crise des opioïdes en Amérique du Nord ». Ce sujet en est un de grande importance pour les internistes. En effet, dans nos cliniques ou à l'hôpital, nous rencontrons fréquemment des patients qui ont des problèmes de dépendance aux opioïdes et nous nous devons d'avoir une stratégie médicale judicieuse et empreinte de compassion pour prendre en charge ces situations. C'est aussi pour nous l'occasion de contribuer à prévenir un problème qui débute souvent par une prescription médicale.

Certains problèmes de consommation dopiö̈des sont le résultat d'une consommation illicite de drogues, mais bien plus souvent la situation débute par une prescription pour la prise en charge d'une douleur aiguë ou chronique. C'est dans le traitement de la douleur aiguë que les médecins ont le plus de chances de prévenir le développement d'une dépendance aux opioïdes (et des comorbidités associées). Dans le traitement de la douleur aiguë (musculo-squelettique, pleurétique ou post-opératoire) une prescription d’opioïdes ne devrait pas dépasser 3 à 7 jours. Délivrer une ordonnance pour deux semaines d'opioïdes est inutile et expose le patient à des risques de développer un problème de dépendance. Certains patients continueront de prendre des opioïdes au-delà de la première semaine s'ils ont une ordonnance qui le leur permet, même si la douleur n'en justifie pas l'usage. C'est la première étape d'une consommation négligente et d'une dépendance subséquente. Si la douleur aiguë demeure suffisamment sévère pour justifier le maintien d'un traitement aux opioïdes au-delà d'une semaine, l'on devrait réévaluer le patient pour comprendre le pourquoi de la situation.

Dans le cas de problèmes de douleur chronique que les traitements sans opioïdes n’ont pas réussi à apaiser, les opioïdes peuvent être considérés comme un élément de la thérapie.
Toutefois, une escalade de la dose à plus de $50 \mathrm{mg} /$ jour d'équivalent en morphine est peu susceptible d'offrir au patient le soulagement supplémentaire qui justifierait une dose plus forte. Chez un faible pourcentage de patients qui requièrent une dose exceptionnellement élevée de médicament pour bénéficier de l'effet clinique maximal associé à un traitement aux opioïdes, un maximum de $90 \mathrm{mg} /$ jour d'équivalent en morphine peut être nécessaire1. Aller au-delà de cette dose est contestable sur le plan pharmacologique, car le plafond de la courbe de réponse au dosage de l'antalgique aura été atteint et l'augmentation de la dose aura pour seul effet d'accroitre le risque de réactions indésirables. De plus, le problème des opioïdes associé à l'hyperalgésie risque de débuter, ce qui nous conduit à l'analogie du chien qui court après sa queue; la dose continue d'augmenter, mais ne procurera jamais le soulagement attendu. C'est chez ce type de patients qu'il faut se concentrer sur des mesures qui les aideront à " vivre " avec leur douleur, plutôt que sur un traitement vaguement susceptible de la réduire ou de léliminer. Il n'y a pas de remède à la douleur chronique, mais il existe des interventions autres que le recours aux opioïdes pour améliorer la qualité de vie d'un patient.

Nous pouvons contribuer à corriger le problème des opioïdes si nous adoptons deux comportements en matière de prescription. Dans la prise en charge de la douleur aiguë, on limitera le recours aux opioïdes à une durée de 3 à 7 jours. Quant à la douleur chronique, elle devrait être traitée avec un maximum de $90 \mathrm{mg}$ /jour d'équivalent en morphine (de préférence $50 \mathrm{mg}$ ), en association avec des thérapies complémentaires qui aident le patient à se concentrer sur l'amélioration de sa qualité de vie, malgré la douleur persistante.

\section{Référence}

1. BUSSE, J.W., S. Craigie, D.N. Juurlink, et coll. « Guideline for opioïdes therapy and chronic noncancer pain », CMAJ, vol. 189, no 18 (8 mai 2017), p. E659-E666; DOI: 10.1503/cmaj.170363.

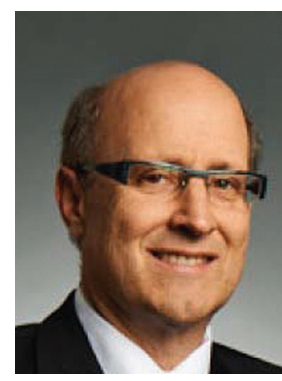

